University of Nebraska - Lincoln

DigitalCommons@University of Nebraska - Lincoln

April 2007

\title{
Slow turning reveals enormous quadrupolar interactions (STREAQI)
}

John Persons

University of Nebraska - Lincoln

Gerard S. Harbison

University of Nebraska - Lincoln, gharbison1@unl.edu

Follow this and additional works at: https://digitalcommons.unl.edu/chemistryharbison

Part of the Chemistry Commons

Persons, John and Harbison, Gerard S., "Slow turning reveals enormous quadrupolar interactions (STREAQI)" (2007). Gerard Harbison Publications. 5.

https://digitalcommons.unl.edu/chemistryharbison/5

This Article is brought to you for free and open access by the Published Research - Department of Chemistry at DigitalCommons@University of Nebraska - Lincoln. It has been accepted for inclusion in Gerard Harbison Publications by an authorized administrator of DigitalCommons@University of Nebraska - Lincoln. 
Published in Journal of Magnetic Resonance 186 (2007), doi:10.1016/j.jmr.2007.03.002 Copyright (C 2007 Elsevier Inc. Used by permission. http://www.sciencedirect.com/science/journal/10907807

Submitted December 18, 2006; revised March 1, 2007; published online March 12, 2007.

\title{
Communication
}

\section{Slow turning reveals enormous quadrupolar interactions (STREAQI)}

\author{
John Persons and Gerard S. Harbison \\ Department of Chemistry, University of Nebraska-Lincoln, Lincoln, NE 68588-0304
}

\begin{abstract}
We introduce a new solid-state NMR method, which uses very slow sample rotation to visualize NMR spectra whose width exceeds feasible spectrometer bandwidths. It is based on the idea that if we reorient a tensor by a known angle about a known axis, the shifts in the NMR frequencies observed across the spectral width allow us to reconstruct the entire tensor. Called STREAQI (Slow Turning Reveals Enormous Anisotropic Quadrupolar Interactions), this method allows us to probe NMR nuclei that are intractable to current methods. To prove the concept and demonstrate its promise we have implemented the method for several ${ }^{79} \mathrm{Br}$ containing samples with quadrupolar coupling constants in the range of 10-50 MHz.
\end{abstract}

Keywords: Quadrupolar interactions, Sample rotation, NMR, Bromine-79

Quadrupolar half-integer-spin nuclei account for $68 \%$ of the NMR-active nuclei listed in the Handbook of Chemistry and Physics, and advances in NMR methodology over the last quarter century have made these nuclei more useful probes of the chemical and electrostatic environment in solids. Most experiments observe the central $1 / 2$ to $-1 / 2$ transition of such nuclei, which is perturbed by the nuclear quadrupole coupling only to second (more properly even) orders. Narita et al. [1] and Baugher et al. [2] showed how the singularities of the second-order quadrupole perturbed central transition spectra could be used to determine the principal values of the quadrupole coupling tensor, and Kundla et al. [3] computed how these powder patterns are narrowed but not eliminated under magic angle spinning. The advent of double rotation [4] and [5] dynamic angle spinning [5] and [6], MQMAS [7], and STMAS [8] has provided a toolbox of useful experiments for narrowing these resonances to isotropic lines, and opened up a number of useful applications of highresolution solid-state NMR of quadrupolar nuclei for the study of minerals, glasses, and other systems.
Unfortunately, however, there remains an extensive set of NMR nuclei whose quadrupolar couplings, even at the highest commonly accessible fields, are simply too large to be averaged by modern-line narrowing methods, or even to be contained within the effective bandwidth of modern NMR instruments. In general, such systems are still studied using static samples, and some variant of frequency sweeping. While some improvement in sensitivity has been gained by spikelet spin-echo methods [9], in general these spectra require cumbersome acquisition of multiple free induction decays across the width of the central transition, which may be several $\mathrm{MHz}$, exceeding the effective spectrometer bandwidth by a factor of ten or more. In many respects, these systems represent the last dogged holdouts, resisting the revolution in NMR that began with the implementation of Fourier transform and coherent averaging methods.

The expression for the second order quadrupolar coupling frequency for the central transition independently derived by Narita et al. [1] and by Baugher et al. [2] is given as Eq. (1); we will use the former authors' angle convention: 


$$
\begin{aligned}
v_{\mathrm{Q}}^{(2)}= & \frac{9 C_{\mathrm{Q}}^{2}(2 I+3)}{16 v_{0} I^{2}(2 I-1)} \\
& \times\left[\begin{array}{c}
\left(\frac{27}{8}-\frac{9}{4} \eta \cos 2 \phi+\frac{3}{8} \eta^{2} \cos ^{2} 2 \phi\right) \cos ^{4} \theta \\
+\left(\begin{array}{c}
\left.-\frac{30}{8}+\frac{1}{2} \eta^{2}+2 \eta \cos 2 \phi+\frac{3}{4} \eta^{2} \cos ^{2} 2 \phi\right) \cos ^{2} \theta \\
+\left(\frac{3}{8}-\frac{1}{3} \eta^{2}+\frac{1}{4} \eta \cos 2 \phi+\frac{3}{8} \eta^{2} \cos ^{2} 2 \phi\right)
\end{array}\right]
\end{array}\right.
\end{aligned}
$$

with the quadrupole coupling constant $C_{\mathrm{Q}}=e Q V_{z z}$ and the Zeeman frequency $v_{0}=\gamma B_{0}$. This formula makes clear the obvious relation between the quadrupolar-shifted transition frequency and the polar angles $\theta, \varphi$, which define the direction of magnetic field in the frame of reference of the electric field gradient (EFG) at the nuclear site; as usual, $V_{z z}$ and $\eta$ are the largest principal value of the EFG tensor, and the asymmetry parameter of that tensor, respectively. Thus, the second-order shift is directly dependent on the orientation of the EFG with respect to the field. Conversely, therefore, if one can measure the shift in the NMR frequency as a result of a defined change in EFG orientation, at more than one point in the spectrum, one can in principle infer the magnitudes of $C_{\mathrm{Q}}$ and $\eta$.

Possible methods for changing the angles $\theta$ and $\varphi$ include a discontinuous jump between two orientations, such has been used in dynamic-angle spinning [5] and [6] and magic-angle hopping [10]; a field jump, implemented electronically; or slow continuous sample rotation over an extended interval, as has been implemented in the magic-angle turning experiment [11] and extensions of it [12]. We have adopted the last method, largely for practical reasons: slow rotation is readily achieved using a magic-angle spinning probe; it can be carefully and precisely controlled without the wear-and-tear that results from fast, discrete jumps, and does not require shifting the direction of large magnetic fields. The disadvantage, of course, is that the experiment now departs from the ideal case discussed above, since the sample is now slowly rotating rather than static in the evolution and acquisition dimensions. In principle, the result will be to transform a single-crystallite spectrum in each dimension to a powder pattern, as was observed in the related STEAMER experiment of Hill and Yesinowski [13]; Using numerical methods, we can explicitly include the effect of slow sample rotation in the evolution and acquisition dimensions. However, for heuristic reasons, we shall first consider the "ideal" STREAQI experiment, in the limit of slow reorientation, by reducing the spinning speed and lengthening $t_{\mathrm{s}}$, keeping the angular excursion during $t_{\mathrm{s}}$ constant.

We begin our mathematical analysis of the experiment by defining the orientation of the EFG tensor with respect to the axis of reorientation, which is related to the field by the angle $\zeta$. The Euler angles relating the EFG principal axis system to the rotor frame (rotor axis along $z$; field in $x z$ plane) are $(\alpha, \beta, \gamma)$. If the sample is reoriented by an angle $\delta$ about the rotor axis, the sequence of reorientations taking the quadrupolar spin from the EFG frame to the laboratory frame (field along $z$; rotor axis in $x z$ plane) is $R_{z}(\alpha) R_{y}(\beta) R_{z}(\gamma)$ $R_{z}(\delta) R_{y}(\zeta)$. If we restrict ourselves for the moment to reori- entations about the magic angle $\left(\zeta=54.7^{\circ}\right)$, a little algebra gives us the Narita formula in terms of the new angles.

$$
\begin{aligned}
v_{\mathrm{Q}}^{(2)}= & \frac{C_{\mathrm{Q}}^{2}(2 I+3)}{256 v_{0} I^{2}(2 I-1)} \\
& \times\left[\begin{array}{c}
9-30 A_{3}+9 A_{3}^{2}-2 \eta\left(A_{1}-A_{2}\right)\left(1+3 A_{3}\right) \\
+\eta^{2}\left(-8+\left(A_{1}-A_{2}\right)^{2}+4 A_{3}\right)
\end{array}\right] \\
A_{1}= & {[\sqrt{2}(\sin \alpha \cos \beta \cos \gamma+\cos \alpha \sin \gamma)+\sin \alpha \sin \beta]^{2} } \\
A_{2}= & {[\sqrt{2}(\cos \alpha \cos \beta \cos \gamma-\sin \alpha \sin \gamma)+\cos \alpha \sin \beta]^{2} } \\
A_{3}= & (\cos \beta-\sqrt{2} \sin \beta \cos \gamma)^{2}
\end{aligned}
$$

entation. After the reorientation, the frequency is obtained with the same formula by replacing $\gamma$ with $(\gamma+\delta)$.

We have implemented a method for measuring this orientation-dependent shift using a standard three pulse sequence used in the EXSY/NOESY experiment [14]

$90^{\circ}-t_{1}-90^{\circ}-t_{\mathrm{s}}-90^{\circ}-t_{2}$

in which the central transition is excited, allowed to precess during $t_{1}$, and alternate components of the phase-encoded magnetization stored along $z$ using a TPPI based scheme. Following a delay $t_{\mathrm{s}}$ in which the sample orientation is changed, the changed frequencies are observed during $t_{2}$ following a third pulse. Two-dimensional Fourier transformation then maps the two frequencies onto each other. Computed spectra using this idealized scheme are presented as surface plots in Fig. 1. Simulations assume a $C_{\mathrm{Q}}=16 \mathrm{MHz}$, $v_{0}=150 \mathrm{MHz}$, an angle jump of $2.4^{\circ}$, and asymmetry parameters of $0,0.2,0.4,0.6,0.8$ and 1.0 . We assume the jump is about the magic-angle; this is not an intrinsic requirement of the method, but allows the computations to be related to our experimental data, which were for practical reasons carried out in a standard MAS probe. Computed spectra consist of a series of overlapping ellipse-like ridges, with pronounced singularities at several positions along the diagonal, reminiscent of the two-dimensional exchange spectra reported by Spiess and co-workers [15]. Unlike the exchange case, however, careful mathematical analysis failed to reveal an analytical functional form $f\left(v_{1}, v_{2}\right)$ for the ridges.

Figs. 2(a) and (c) compare ideal and experimental STREAQI spectra of crystalline tyrosine hydrobromide, prepared by cooling a hot saturated solution of tyrosine in excess $2 \mathrm{M}$ hydrobromic acid. $C_{\mathrm{Q}}$ and $\eta$ were obtained by fitting the $1 \mathrm{D}$ powder pattern, giving $C_{\mathrm{Q}}=11.26 \mathrm{MHz}(\eta=0.86)$, although it appears that in this sample there is a small chemical shielding anisotropy that affects the lineshape and positions of the singularities slightly. The two-dimensional STREAQI spectrum, obtained with the same $90^{\circ}$ pulse length, a spinning speed of $300 \mathrm{~Hz}$ about the magic angle, a $t_{\mathrm{s}}$ delay of $100 \mu \mathrm{s}$, corresponding to an angle excursion of $10.8^{\circ}$, and two-dimensional hypercomplex acquisition and processing [16], gave the spectrum shown. There is reasonably good correspondence 

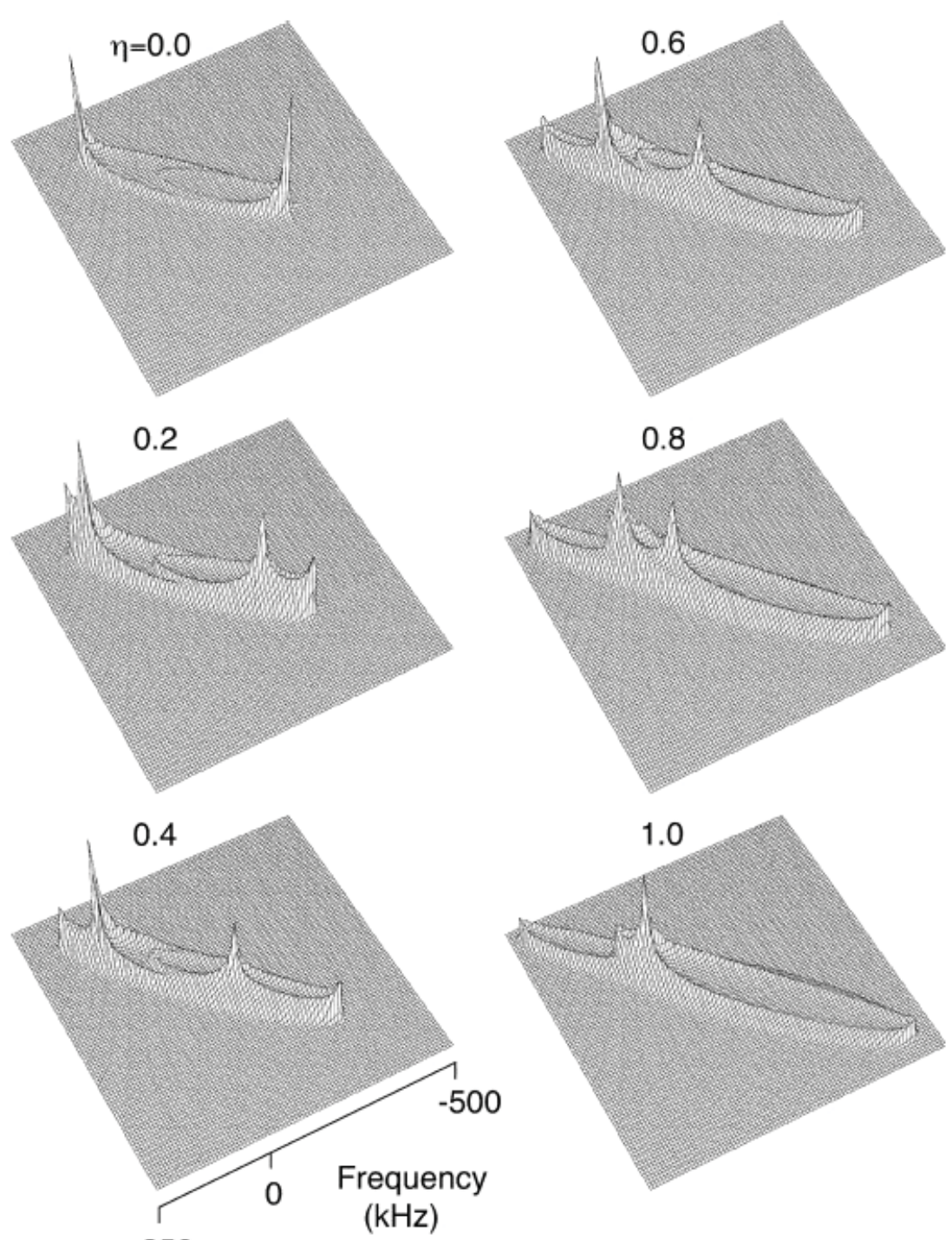

250

Fig. 1. Computed STREAQI spectra of a hypothetical substance with $C_{\mathrm{Q}}=16 \mathrm{MHz}, v_{0}=150 \mathrm{MHz}$, in the limit of slow-spinning at the magic angle, with a $2.4^{\circ}$ jump during $t_{\mathrm{s}}$.
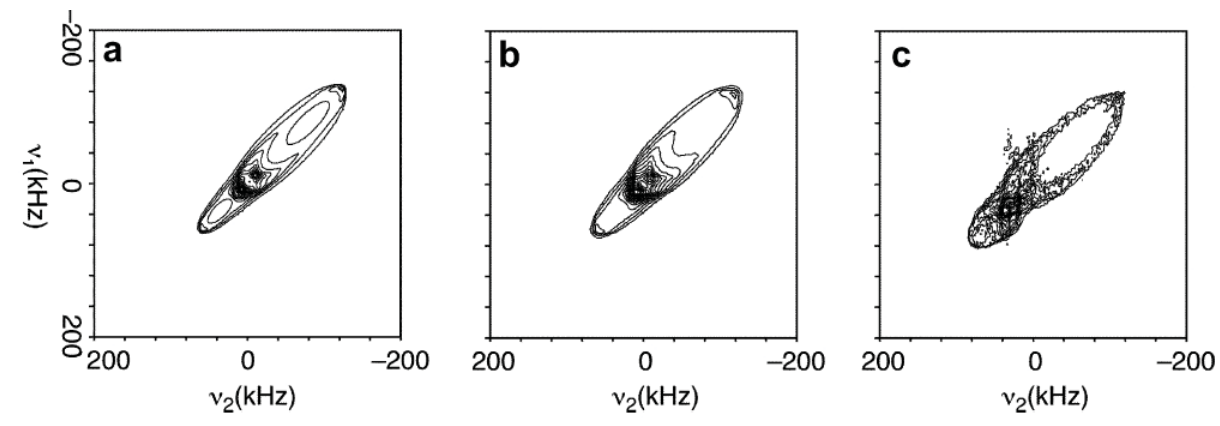

Fig. 2. ${ }^{79} \mathrm{Br}$ STREAQI spectra of 1-tyrosine hydrobromide, simulated using the assumption (a) of a discrete jump between two static orientations and (b) continuous sample rotation; compared with (c) an experimental spectrum, obtained as described in the text.

with the computed spectrum, with the same pattern of singularities and pseudo-elliptical ridges.

In order to ascertain whether the differences between experiment and theory could be ascribed to the approxi- mation of discrete jumps between static spectra in $t_{1}$ and $t_{2}$, we computed STREAQI spectra using the same parameters, but assuming continuous sample rotation during $t_{1}$ and $t_{2}$, using a fourth-order Runge-Kutta algorithm with 
$0.5 \mu$ s time increments to numerically evaluate the evolution of the spin system in the presence of a slowly rotating quadrupolar Hamiltonian. The result of such a simulation is shown in Fig. 2(b), The agreement between simulated and experimental spectra is somewhat improved; in particular, the width of the pattern orthogonal to the main diagonal is increased in the continuous rotation simulation, compared with the discrete-jump simulation. Nonetheless, the simulated spectrum is qualitatively unchanged.

The sequence, however, likely to be most useful in circumstances where the second order pattern width is many times the spectrometer bandwidth. This is the case for leucine hydrobromide at $14 \mathrm{~T}$, whose pure NQR frequency has been reported [17] to be $25.35 \mathrm{MHz}$ at $27^{\circ} \mathrm{C}$. Ordinary 1D NQR does not give an asymmetry parameter. However, the spin $3 / 2 \mathrm{NQR}$ frequency

$$
v_{\mathrm{Q}}=\frac{C_{\mathrm{Q}}}{2}\left(1+\frac{\eta^{2}}{3}\right)^{\frac{1}{2}}
$$

is a weak function of $\eta$. Since $\eta$ is likely to be similar to that of the isomorphous hydroiodide $(\eta=0.66)$, we expect a $C_{\mathrm{Q}}$ of around $50 \mathrm{MHz}$, relatively insensitive to error in $\eta$. These two parameters are a good starting point for simulations.

Fig. 3(c) shows the STREAQI spectrum of a sample of crystalline leucine hydrobromide, obtained by the same method used to prepare tyrosine hydrobromide, and with a spinning speed of $300 \mathrm{~Hz}$, but with a STREAQI delay of $20 \mu \mathrm{s}$. This is clearly just a very small part of a $2 \mathrm{D}$ spectrum that extends over several MHz. Nonetheless, a clear pseudoellipse, and a diagonal spine, ending in a singularity, are observed. Examination of simulated STREAQI patterns with $C_{\mathrm{Q}}$ and $\eta$ values similar to those expected indicate that the frequencies $v_{3}$ and $v_{4}$, computed by Baugher et al. [2] as

$$
v_{3}=\frac{C_{\mathrm{Q}}^{2} \eta^{2}}{48 v_{0}}
$$

likely correspond to the singularity, and the extremum of the ellipse, respectively. Using these two frequencies $v_{3}=116 \mathrm{kHz}$ and $v_{4}=434 \mathrm{kHz}$, we obtained an estimated $C_{\mathrm{Q}}$ frequency of $49.0 \mathrm{MHz}$ and an asymmetry parameter of 0.59 . Using Eq. (3) we compute a pure NQR frequency of $25.88 \mathrm{MHz}$, in good agreement with the experimental $300 \mathrm{~K}$ value of $25.35 \mathrm{MHz}$. It can be seen from Fig. 3(a), however, that the simulated STREAQI spectrum, using the ideal model, no longer gives a good fit to experimental data; clearly, the assumption of static evolution and detection periods spaced by an instantaneous jump breaks down for such large quadrupolar couplings. The spectrum simulated with continuous rotation (Fig. 3 (b)) is considerably improved, but agreement with the experimental data is still by no means perfect. This is unsurprising: the simulation does not allow for finite bandwidth or evolution during the pulses, which is likely to be a problem off-resonance. It is also likely that the implicit assumption of an isolated central transition breaks down for large quadrupole couplings, and the evolution of the full $4 \times 4$ density matrix needs to be explicitly simulated. However, the location of the singularities is unchanged by these effects, and therefore a single on-resonance STREAQI pattern, or in worst cases two STREAQI patterns at different frequencies, should be adequate to determine the quadrupolar tensor magnitudes of materials whose spectral breadth greatly exceeds the spectrometer bandwidth.

Our analysis implicitly assumes the chemical shielding anisotropy is negligibly small; given the formally spherical bromide cation, it seems to be a reasonable assumption in the present instance, and certainly we find no evidence of a significant dispersion of isotropic or anisotropic shifts in the amino-acid hydrobromides. Inclusion of a comparable chemical shielding tensor, of course, considerably complicates the analysis. However, given the different angular dependence of the chemical shift and quadrupolar tensors, it may be possible to distinguish their influence by obtaining STREAQI spectra at different rotation angles, In fact, the $90^{\circ}$ STREAQI experiment (rotating normal to the field), as
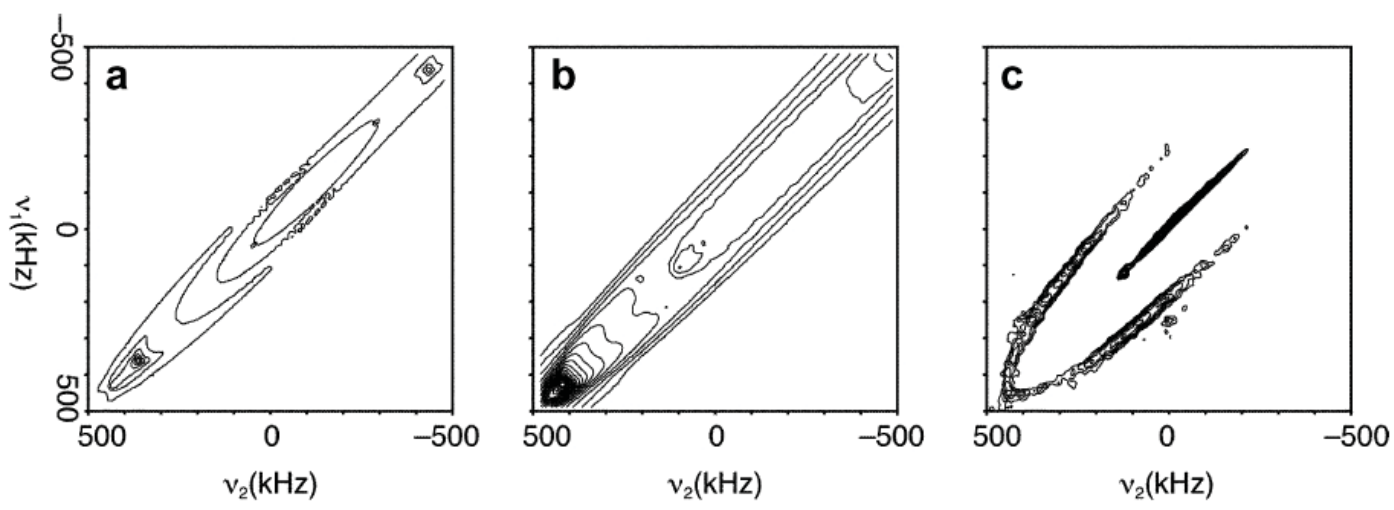

Fig. 3. ${ }^{79} \mathrm{Br}$ STREAQI spectra of 1-leucine hydrobromide, simulated using the assumption of (a) a discrete jump between two static orientations and (b) continuous sample rotation; compared with (c) an experimental spectrum, obtained as described in the text. 
was done in STEAMER [13] and in an EPR experiment by Sierra and Schweiger [18] poses some considerable theoretical advantages over magic-angle STREAQI, both in terms of sensitivity and the simplicity of the spectra. Consideration of this experiment must, however, await further research.

\section{Acknowledgment}

This work was funded by a grant from NIH (R01 GM 065252).

\section{References}

[1] K. Narita, J. Umeda and H. Kusumoto, Nuclear magnetic resonance powder patterns of the second-order nuclear quadrupole interaction in solids with asymmetric field gradient, J. Chem. Phys. 44 (1966), pp. 2719-2723.

[2] J.F. Baugher, P.C. Taylor, T. Oja and P.J. Bray, Magnetic resonance powder patterns in the presence of completely asymmetric quadrupole and chemical shift effects: Application to metavanadates, J. Chem. Phys. 50 (1969), pp. 4914-4925.

[3] E. Kundla, A. Samoson and E. Lippmaa, High-resolution NMR of quadrupolar nuclei in rotating solids, Chem. Phys. Lett. 83 (1981), pp. 229-232.

[4] A. Samoson, E. Lippmaa and A. Pines, High-resolution solidstate NMR. Averaging of second-order effects by means of a double-rotor, Mol. Phys. 65 (1988), pp. 1013-1018.

[5] A. Llor and J. Virlet, Towards high-resolution NMR of more nuclei in solids: sample spinning with time-dependent spinner axis angle, Chem. Phys. Lett. 152 (1988), pp. 248-253.

[6] K.T. Mueller, B.Q. Sun, G.C. Chingas, J.W. Zwanziger, T. Terao and A. Pines, Dynamic-angle spinning of quadrupolar nuclei, J. Magn. Reson. 86 (1990), pp. 470-487.

[7] A. Medek, J.S. Harwood and L. Frydman, Multiple-quantum magic-angle spinning NMR: A new method for the study of quadrupolar nuclei in solids, J. Am. Chem. Soc. 117 (1995), pp. 12779-12787.
[8] Z. Gan, Isotropic NMR spectra of half-integer quadrupolar nuclei using satellite transitions and magic-angle spinning, J. Am. Chem. Soc. 122 (2000), pp. 3242-3243.

[9] F.H. Larsen, H.J. Jakobsen, P.D. Ellis and N.C. Nielsen, Sensitivity-enhanced quadrupolar-echo NMR of half-integer quadrupolar nuclei. Magnitudes and relative orientation of chemical shielding and quadrupolar coupling tensors, J. Phys. Chem. A 101 (1997), pp. 8597-8606.

[10] A. Bax, N.M. Szeverenyi and G.E. Maciel, Correlation of isotropic shifts and chemical shift anisotropies by two-dimensional Fourier-transform magic-angle hopping NMR spectroscopy, J. Magn. Reson. 52 (1983), pp. 147-152.

[11] Z. Gan, High-resolution chemical shift and chemical shift anisotropy correlation in solids using slow magic angle spinning, J. Am. Chem. Soc. 114 (1992), pp. 8307-8309.

[12] J.Z. Hu, D.W. Alderman, R.J. Pugmire and D.M. Grant, A high-resolution 3D separated-local-field experiment by means of magic-angle turning, J. Magn. Reson. 126 (1997), pp. 120 126.

[13] A. Hill and J.P. Yesinowski, A slow-turning method for measuring large anisotropic interactions in inhomogeneously broadened nuclear magnetic resonance spectra, J. Chem. Phys. 106 (1997), pp. 8650-8659.

[14] J. Jeener, B.H. Meier, P. Bachmann and R.R. Ernst, Investigation of exchange processes by two-dimensional NMR spectroscopy, J. Chem. Phys. 71 (1979), pp. 4546-4553.

[15] C. Schmidt, B. Blümich and H.W. Spiess, Deuteron two-dimensional exchange NMR in solids, J. Magn. Reson. 79 (1988), pp. 269-290.

[16] D.J. States, R.A. Haberkorn and D.J. Ruben, A two-dimensional nuclear Overhauser experiment with pure absorption phase in four quadrants, J. Magn. Reson. 48 (1982), pp. 286-292.

[17] S. Fleck and A. Weiss, Hydrogen bonds in hydrohalides of amino acids. A ${ }^{79,81} \mathrm{Br}$ and ${ }^{127} \mathrm{I}$ NQR study, Ber. Bunsengesell. Phys. Chem. 88 (1984), pp. 956-963.

[18] G.A. Sierra and A. Schweiger, Right-angle sample spinning electron spin resonance, Rev. Sci. Instr. 68 (1997), pp. 13161323. 